\title{
Life-cycle Asset Allocation and Optimal Consumption Using Stochastic Linear Programming
}

\begin{abstract}
We consider optimal consumption and (strategic) asset allocation of an investor with uncertain lifetime. The problem is solved using a multi-stage stochastic linear programming (SLP) model to be able to generalize the closed-form solution obtained by Richard (1975). We account for aspects of the application of the SLP approach which arise in the context of life-cycle asset allocation, but are also relevant for other problems of similar structure. The objective is to maximize the expected utility of consumption over the lifetime and of bequest at the time of death of the investor. Since we maximize utility (rather than other objectives which can be implemented more easily) we provide a new approach to optimize the breakpoints required for the linearization of the utility function. The uncertainty of the problem is described by discrete scenario trees. The model solves for the rebalancing decisions in the first few years of the investor's lifetime, accounting for anticipated cash flows in the near future, and applies Richard's closed-form solution for the long, subsequent steady-state period. In our numerical examples we first show that available closed-form solutions can be accurately replicated with the SLP-based approach. Second, we add elements to the problem specification which are usually beyond the scope of closed-form solutions. We find that the SLP approach is well suited to account for these extensions of the classical Merton setting.
\end{abstract}

JEL classification codes: C61, G11 


\section{Introduction}

One of the classical problems in finance is the optimal consumption and asset allocation over the life-cycle of a finitely-lived investor. This problem lies at the heart of the subfield of personal finance, and financial advisors as well as portfolio and pension fund managers throughout the world are faced with it every day. The interest and activities in this research area have grown in recent years, partly spurred by growing worldwide concern about the stability of public pension systems and the resulting trend towards private pensions. Classical treatments of this problem are Samuelson (1969) and Merton $(1969,1971)$ who formulate models in settings which allow for closed-form solutions. Since then various models in more general settings (e.g. accounting for labor income or time-varying investment opportunities) have been proposed, many of which require to use numerical solution techniques.

We use multi-period stochastic linear programming (SLP) to solve the problem of optimal life-cycle asset allocation and consumption. The method has been explicitly chosen with the practical application of our approach in mind. This distinguishes our work from literature which focuses more on gaining general insights into the dependencies between investment/consumption decisions and state variables but excludes a number of (possibly important) real-world aspects on purpose to preserve analytical tractability. For example, many models are confined to a small number of risky assets (often only one), do not allow for constraints on the asset allocation, ignore transactions costs, or cannot take exogenous cash flows into account. In contrast, many of these features which are considered important for investment decisions in practice can be easily incorporated when using SLP. Combined with the availability of efficient solvers, this explains why the SLP approach has been successfully applied to a wide range of problems (see e.g. Ziemba and Mulvey 1998, Wallace and Ziemba 2005), in particular in the context of asset-liability management (see e.g. Cariño et al. 1998, Dempster et al. 2003, Zenios and Ziemba 2006, Geyer and Ziemba 2007), or contingent 
claims analysis (see King 2002).

Key elements of our model are: The expected utility of consumption over the investor's lifetime and expected utility of bequest is maximized, and mortality risk is accounted for. Consumption and investment decisions are optimized jointly, and personal characteristics of the investor can be taken into account (e.g. risk attitude, retirement, future cash flows for major purchases or associated with other life events). In addition to including those aspects another objective is to solve certain (technical) problems which are typically encountered in the formulation and implementation of SLPs for life-cycle asset allocation and consumption problems (e.g. the long planning horizon is in conflict with a manageable problem size).

The present paper is - as far as we know - the first application of stochastic linear programming in a life-cycle asset allocation context. We maximize expected utility rather than other objectives which can be implemented more easily (e.g. piecewise linear or quadratic penalty functions, or minimizing $\mathrm{CVaR}$ ). As far as we know no other papers in this area use utility functions in this way and for that purpose. The accuracy of the solution depends strongly on the way the utility function is linearized. Linearizing such functions can be rather difficult, in particular for high degrees of risk aversion. Therefore it is essential to optimize the breakpoints required for their linearization. We propose and implement a new approach for that purpose and show that closed-form solutions can be replicated with very high precision.

The long time intervals involved in life-cycle asset allocation problems pose a notoriously difficult problem. To keep the dimension of the problem manageable SLP-based approaches typically rebalance the portfolio very infrequently and define consumption plans for rather long time intervals. We provide two contributions to this aspect. First, we incorporate a closed-form solution for optimal subperiod consumption during (long) time intervals in the objective. Second, the model formulation combines decisions for a few short time periods in the near future with a closed-form solution for the long, subsequent steady-state period. Both closed-form solutions are integrated into the SLP 
formulation and take survival probabilities into account.

The SLP approach extends the range of available numerical methods for solving life-cycle problems. ${ }^{1}$ One type of numerical methods works via grid methods discretizing the state space (see e.g. Brennan et al. 1997, Barberis 2000, Cocco et al. 2005, Gomes and Michaelides 2005). Another type derives a system of equations which is solved numerically (see Schroder and Skiadas 1999). Detemple et al. (2003) propose a simulation-based method to approximate deviations from a closed-form solution. Brandt et al. (2005) combine Monte Carlo simulation and regression techniques, and are inspired by the option pricing algorithm of Longstaff and Schwartz (2001). The linear nature of the SLP approach bears some similarities to Campbell et al. (2003) who linearize key problem elements (e.g. the budget constraint, and the Euler equation).

The analysis of return predictability and its impact on asset allocation decisions has found considerable attention in the literature (see e.g. Barberis 2000, Campbell et al. 2003, Brandt et al. 2005). For that purpose, a number of aspects (e.g. mortality risk, constraints on asset weights, transaction costs, labor income or other cash flows) may have to be excluded from the analysis. However, in this paper we rather want to focus on those practically relevant aspects. Therefore we maintain the i.i.d. assumption and extend the Merton-Samuelson-Richard setting along these lines.

The paper is organized as follows: Section 2 describes the stochastic programming model, in particular the formulation of the objective, the optimization approach for its linearization, and the generation of scenarios. In Section 3 results from the SLP are compared to cases where closed-form solutions are available, and results from life-cycle consumption and asset allocation decisions in a more realistic setting are presented. Section 4 concludes.

\footnotetext{
${ }^{1} \mathrm{~A}$ more comprehensive comparison with other numerical methods is very demanding and not the objective of this paper.
} 


\section{Model description}

We consider the consumption and investment decisions of an investor with uncertain lifetime. We start by formulating a generic stochastic linear program to introduce the basic idea of this approach. Subsequently, we introduce our notation and define key variables, formulate the constraints and the objective function. Thereafter, we describe the choice of breakpoints and the scenario generation.

\subsection{Stochastic linear programming}

A two-stage stochastic linear program (SLP) with recourse has the following structure: The problem consists of a first-stage decision $\mathbf{x}_{0}$ with uncertain consequences $\omega_{1}$ (e.g. the uncertain returns associated with an investment). The decisions taken in the second stage $\mathbf{x}_{1}\left(\mathbf{x}_{0}, \omega_{1}\right)$ can be viewed as revisions of the first-stage decisions given realized outcomes of $\omega_{1}$. The objective is to maximize the utility (or another valuation function) associated with $\mathbf{x}_{0}$ and the expected utility of $\mathbf{x}_{1}\left(\mathbf{x}_{0}, \omega_{1}\right)$ subject to linear constraints relevant in $t=0$ and $t=1$.

$$
\begin{aligned}
& f_{0}\left(\mathbf{x}_{0}\right)+\mathrm{E}\left[f_{1}\left(\mathbf{x}_{1}\left(\mathbf{x}_{0}, \omega_{1}\right)\right)\right] \longrightarrow \max \\
& \text { s.t. } \quad \mathbf{A}_{0} \mathbf{x}_{0} \leq \mathbf{b}_{0} \quad \mathbf{A}_{1} \mathbf{x}_{1}\left(\mathbf{x}_{0}, \omega_{1}\right) \leq \mathbf{b}_{1} .
\end{aligned}
$$

The probability distribution of the random variable $\omega_{1}$ is described in terms of a discrete number of outcomes (so-called scenarios). This implies that $\mathbf{x}_{1}$ represents decisions for each possible scenario. It further implies that the expectation in the objective is evaluated in terms of a sum across outcomes. Accordingly, the constraints in the second stage must hold for each scenario. An important assumption is that randomness is exogenous and is not affected by decisions.

In our application we consider non-linear objective functions. To be able to use linear programming solvers those functions need to be linearized. For that purpose 
a function $f(x)$ is approximated by $m$ linear segments between the breakpoints $b_{j}$ $(j=0, \ldots, m)$. The choice of $b_{j}$ is explained in detail in Section 2.5. The argument $x$ is defined in terms of non-negative decision variables $v_{j}$ associated with each segment:

$$
\begin{aligned}
& x=\sum_{j=0}^{m+1} v_{j} \\
& 0 \leq v_{0} \leq b_{0} \quad v_{m+1} \geq 0 \\
& 0 \leq v_{j} \leq b_{j}-b_{j-1} \quad j=1, \ldots, m .
\end{aligned}
$$

The slopes of the linear segments are given by

$$
\Delta_{j}=\frac{f\left(b_{j}\right)-f\left(b_{j-1}\right)}{b_{j}-b_{j-1}}
$$

and $f(x)$ is approximated by

$$
f(x) \approx \Delta_{1} v_{0}+\sum_{j=1}^{m} \Delta_{j} v_{j}+\Delta_{m} v_{m+1} .
$$

The two-stage formulation of an SLP can be extended to $T$ stages. In general, the probability distribution associated with $\omega_{t}$ depends on the outcomes of the random variable $\omega_{t-1}$. This can be described by a scenario tree (see Figure 1) which is defined by the number of stages and the number of arcs leaving nodes at a particular stage (the branching factor $n_{t}$ ). The tree always starts with a single node which corresponds to the present stage $(t=0)$. Each outcome in $t-1$ is the root of the corresponding sub-tree consisting of $n_{t}$ outcomes in $t$.

Decisions are made at each node of the tree. Non-anticipatory constraints are imposed to guarantee that a decision made at a specific node is identical for all scenarios leaving that node. A single scenario $s_{t}$ is a trajectory that corresponds to a unique path leading from the single node at $t=0$ to a single node at $t$. Two scenarios $s_{t}^{\prime}$ and 
Figure 1: Scenario tree with node structure $2 \times 2 \times 3$

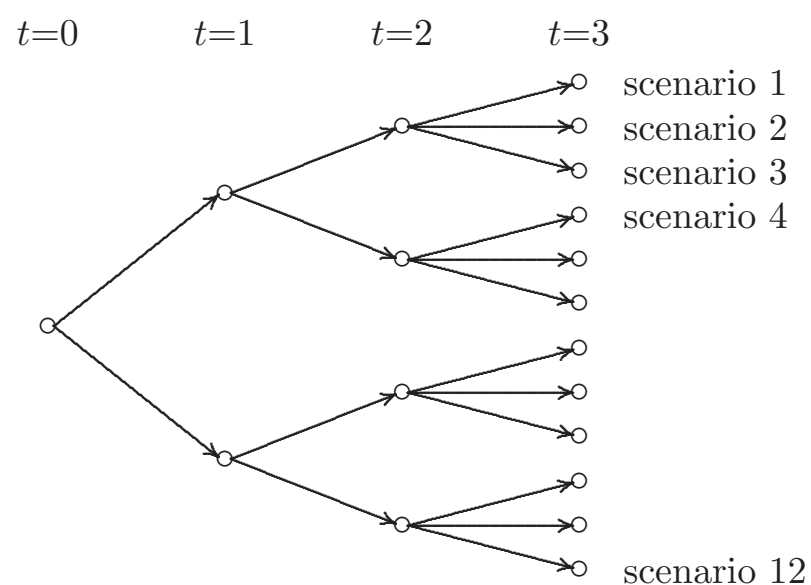

$s_{t}^{\prime \prime}$ are identical until $t$ and differ in subsequent stages $t+1, \ldots, T$. Thus, decisions in $t$ depend on the history of the random variable $\left(\omega_{1}, \ldots, \omega_{t}\right)$, and the decisions taken in all previous stages, which is denoted by $\tilde{\mathbf{x}}_{t}$. In general, a multi-stage stochastic linear program can be formulated as

$$
f_{0}\left(\mathbf{x}_{0}\right)+\sum_{t=1}^{T} \mathrm{E}\left[f_{t}\left(\tilde{\mathbf{x}}_{t}\right)\right] \longrightarrow \max
$$

$$
\text { s.t. } \quad \mathbf{A}_{t} \tilde{\mathbf{x}}_{t} \leq \mathbf{b}_{t} \quad t=0, \ldots, T \text {. }
$$

Given the discrete representation of the random variables and the associated structure of the scenario tree, variables and constraints for each stage and each node of the tree are defined. This typically leads to a large-scale linear program. Since all uncertainty has been converted into corresponding model elements, this representation is called the deterministic equivalent of the SLP. As most of the coefficients in the associated constraint matrices $\mathbf{A}_{t}$ are zero, and the non-zero coefficients follow a special structure, various algorithms to exploit these facts have been proposed (see Birge and Louveaux 1997). Publicly available software to solve large stochastic programming problems is detailed in Wallace and Ziemba (2005). We use routines from the open 
source project COIN-OR (see http://www.coin-or.org). The problem is formulated using the Stochastic Mathematical Programming System (SMPS) input format for multi-stage stochastic programs (see Gassmann and Schweitzer 2001, King et al. 2005) in terms of three input files: the core-, stoch- and time-files. The core-file contains information about the decisions variables, constraints, right-hand-sides and bounds. It contains all fixed coefficients and dummy entries for random elements. The stoch-file reflects the node structure of the scenario tree and contains all random outcomes (i.e. asset returns). The time-file assigns decision variables and constraints to stages. The solution of a typical problem we consider in Section 3 with four stages, three assets, 216 scenarios and 40 breakpoints requires a few seconds using a Pentium 4/640 processor with $3.2 \mathrm{GHz}$ and $1 \mathrm{~GB}$ RAM.

\section{$2.2 \quad$ Variables}

We now introduce our notation and key variables. $N$ is the number of assets the investor can choose from. $t$ denotes stages (points in time) and runs from $t=0$ (now) to $t=T$. $T$ is the number of time intervals. $\tau_{t}$ is the number of years between stage $t$ and stage $t+1$ and the total number of years covered (the planning horizon) is given by $\tau=\tau_{0}+\cdots+\tau_{T-1}$. Given the current age of the investor we define the planning horizon such that his maximum age is 101 years (the mortality tables we use assign a conditional probability of $100 \%$ that a person dies between age 100 and 101). The choice of the length of the time intervals $\tau_{t}$ is described in Section 2.6.

The following (decision) variables are used in the model formulation:

$C_{0} \geq 0, \tilde{C}_{t} \geq 0(t=1, \ldots, T-1) \ldots$ consumption in $t$; e.g. $\tilde{C}_{2}$ is the amount set aside in $t=2$ for consumption between $t=2$ and $t=3$.

$\tilde{R}_{t}^{i}(t=1, \ldots, T ; i=1, \ldots, N) \ldots$ gross return of asset $i$ for the period that ends in $t$. $P_{0}^{i} \geq 0, \tilde{P}_{t}^{i} \geq 0(t=1, \ldots, T-1 ; i=1, \ldots, N) \ldots$ amount of asset $i$ purchased in $t$. $S_{0}^{i} \geq 0, \tilde{S}_{t}^{i} \geq 0(t=1, \ldots, T-1 ; i=1, \ldots, N) \ldots$ amount of asset $i$ sold in $t$. 
$q_{p}^{i}$ and $q_{s}^{i} \ldots$ transaction costs for purchases and sales of asset $i$.

$W_{0}^{i}, \tilde{W}_{t}^{i}(t=1, \ldots, T-1 ; i=1, \ldots, N) \ldots$ total amount invested in asset $i$ in $t$; e.g. $\tilde{W}_{2}^{i}$ is the amount invested in asset $i$ in $t=2$; in $t=3$ the value of this investment will be $\tilde{W}_{2}^{i} \tilde{R}_{3}^{i}$.

$w_{0}^{i} \ldots$ initial value of asset $i$ (before transactions).

$\tilde{B}_{t} \geq 0(t=1, \ldots, T) \ldots$ bequest in $t$ given by $\tilde{B}_{t}=\sum \tilde{R}_{t}^{i} \tilde{W}_{t-1}^{i}$.

$\tau_{t}(t=0, \ldots, T-1) \ldots$ the number of years between stage $t$ and stage $t+1$.

$\varphi_{y} \ldots$ the (conditional) probability to survive the year following year $y$.

$\Phi\left(y_{t}, \tau_{t}\right) \ldots$ the probability to survive the period of length $\tau_{t}$ starting at stage $t$ at an age of $y_{t}$ years; $\Phi\left(y_{t}, \tau_{t}\right)=\prod_{k=y_{t}}^{y_{t}+\tau_{t}-1} \varphi_{k}$.

$\Lambda_{t}(t=1, \ldots, T-1) \ldots$ the probability to be alive at stage $t$ (at an age of $\left.y_{t}\right) ; \Lambda_{t}=\prod_{k=0}^{t-1} \Phi\left(y_{k}, \tau_{k}\right)$.

$\Theta_{t}(t=1, \ldots, T) \ldots$ the probability to die between stage $t-1$ and $t ; \Theta_{t}=\Lambda_{t-1}\left[1-\Phi\left(y_{t}, \tau_{t}\right)\right]$.

$L_{t}(t=0, \ldots, T-1) \ldots$ labor income in $t$; e.g. $L_{2}$ is the present value of labor income received between $t=2$ and $t=3$.

$F_{t}(t=0, \ldots, T-1) \ldots$ fixed cash flow paid or received in $t$; e.g. $F_{2}$ is the present value of cash flows paid or received between $t=2$ and $t=3$.

$r$... the risk-free interest rate.

$\delta \ldots$ the investor's time preference rate, $d=\exp \{-\delta\}$ is the time discount factor, and $D_{t}$ is the time discount factor applicable at stage $t$ :

$$
D_{t}=\exp \left\{-\delta \sum_{i=0}^{t-1} \tau_{i}\right\}
$$

The stochastic returns $\tilde{R}_{t}^{i}$ describe the uncertainty faced by the investor. The procedure to simulate their values and to construct the scenario tree is described in Section 2.6. $C_{0}, \tilde{C}_{t}, W_{0}^{i}, \tilde{P}_{t}^{i}, \tilde{S}_{t}^{i}, \tilde{W}_{t}^{i}$ and $\tilde{B}_{t}$ are the decision variables of the problem and their values are obtained from the optimal solution of the stochastic linear program. 
Labor income is computed on the basis of initial labor income $\mathcal{L}_{0}$, the annual labor growth rate $\ell$, the number of years until retirement $y_{r}$, and the fraction of income during retirement $f_{r}$. The annual stream of income before retirement is given by (the index $y$ denotes years $) \mathcal{L}_{y}=\mathcal{L}_{0} \exp \{y \ell\}\left(y=1, \ldots, y_{r}\right)$ and by $\mathcal{L}_{y}=f_{r} \mathcal{L}_{y_{r}} \exp \left\{\left(y-y_{r}\right) \ell_{r}\right\}$ $\left(y=y_{r}+1, \ldots, \tau\right)$ after retirement, where $\ell_{r}$ is the growth rate of labor income after retirement. The present value of labor income used in the budget constraints (see below) is defined as

$$
L_{t}=\sum_{y=j_{t}}^{k_{t}} \mathcal{L}_{y}\left[\left(1-\Phi\left(y_{t}, y-1\right)\left(1-\varphi_{y}\right)\right) \exp \left\{-r\left(y-j_{t}+1\right)\right\}\right]
$$

where

$$
j_{t}=1+\sum_{i=0}^{t-1} \tau_{i} \quad k_{t}=j_{t}+\tau_{t}-1
$$

$\Phi\left(y_{t}, y-1\right)$ is the probability to survive until the beginning of year $y$ given age $y_{t}$ at stage $t$, and $\left(1-\varphi_{y}\right)$ is the probability to die in the subsequent year. Labor income $\mathcal{L}_{y}$ is thus reduced by an amount that corresponds to the premium of a fairly priced life insurance (see Richard 1975).

\subsection{Constraints}

The budget equations are given by

$$
\begin{aligned}
& C_{0}+\sum_{i=1}^{N} P_{0}^{i}\left(1+q_{p}^{i}\right)=\sum_{i=1}^{N} S_{0}^{i}\left(1-q_{s}^{i}\right)+L_{0}+F_{0} \\
& \tilde{C}_{t}+\sum_{i=1}^{N} \tilde{P}_{t}^{i}\left(1+q_{p}^{i}\right)=\sum_{i=1}^{N} \tilde{S}_{t}^{i}\left(1-q_{s}^{i}\right)+L_{t}+F_{t} \quad t=1, \ldots, T-1 .
\end{aligned}
$$


The value of investments accumulates according to the following equations:

$$
\begin{aligned}
& W_{0}^{i}=w_{0}^{i}+P_{0}^{i}-S_{0}^{i} \quad i=1, \ldots, N \\
& \tilde{W}_{t}^{i}=\tilde{R}_{t}^{i} \tilde{W}_{t-1}^{i}+\tilde{P}_{t}^{i}-\tilde{S}_{t}^{i} \quad t=1, \ldots, T-1, \quad i=1, \ldots, N \\
& \tilde{W}_{T}^{i}=\tilde{R}_{T}^{i} \tilde{W}_{T-1}^{i} \quad i=1, \ldots, N .
\end{aligned}
$$

To model restrictions on the portfolio composition we use the constraints

$$
l_{i} \leq \frac{\tilde{W}_{t}^{i}}{\sum_{i=1}^{N} \tilde{W}_{t}^{i}} \leq u_{i} \quad t=0, \ldots, T-1
$$

where $u_{i}$ is the maximum and $l_{i}$ the minimum weight of asset $i$ in the portfolio. Short sales can be excluded by $l_{i}=0$ or limited by setting $l_{i}$ equal to minus the maximum leverage of asset $i$. In general the decision variables $\tilde{W}_{t}^{i}$ can become negative. However, total wealth must be positive in all periods:

$$
\sum_{i=1}^{N} \tilde{W}_{t}^{i} \geq 0 \quad t=0, \ldots, T
$$

\subsection{Objective}

The objective is to maximize the expected utility of consumption over the lifetime and of bequest at the time of death of the investor:

$$
U\left(C_{0}\right)+E\left[\sum_{t=1}^{T} \Theta_{t} U\left(\tilde{B}_{t}\right) D_{t}+\sum_{t=1}^{T-1} \Lambda_{t} U\left(\tilde{C}_{t}\right) D_{t}\right] \longrightarrow \max
$$

$U$ is a power utility function with constant relative risk aversion $\gamma \cdot \Theta_{t}$ is the probability to die between stage $t-1$ and $t$, and $\Lambda_{t}$ is the probability to be alive in $t$.

$C_{0}$ and $\tilde{C}_{t}$ refer to consumption during the subsequent time interval of length $\tau_{t}$ which may be longer than one year. We assume that the amount $\tilde{C}_{t}$ is not consumed at once but in annual parts. Accordingly, the utility of consuming those parts differs 
from the utility of consuming $\tilde{C}_{t}$ at once. As a simple approximation the utility of consumption over a period of length $\tau_{t}$ can be defined as $\tau_{t} U\left(\tilde{C}_{t} / \tau_{t}\right)$. This can be improved by replacing $1 / \tau_{t}$ with an annuity factor. However, we prefer to use a more refined approach which is based on considering the following optimization problem. We derive our solution by considering stage 0 and three subperiods (years) (i.e. $\tau_{0}=3$ ) and generalize later. Assume for the time being that $C_{0}$ is known. We define the optimal annual consumption levels in the three subperiods $c_{0}, c_{1}$ and $c_{2}$ such that the utility of consumption in the period is maximized

$$
U\left(c_{0}\right)+\Phi_{1} \exp \{-\delta\} U\left(c_{1}\right)+\Phi_{2} \exp \{-2 \delta\} U\left(c_{2}\right) \longrightarrow \max
$$

subject to the constraint $c_{0}+\exp \{-r\} c_{1}+\exp \{-2 r\} c_{2}=C_{0}$. To simplify notation in this derivation $\Phi_{j}=\Phi\left(y_{0}, j\right)$ is the conditional probability to survive the next $j$ years (i.e. to be alive at the beginning of subperiod $j+1$ ), given a current age of $y_{0}$. The constraint assumes that $c_{1}$ and $c_{2}$ are invested at the risk-free rate. For power utility $U(c)=c^{1-\gamma} /(1-\gamma)$ we have

$$
\begin{aligned}
L= & \frac{c_{0}^{1-\gamma}}{1-\gamma}+\Phi_{1} \exp \{-\delta\} \frac{c_{1}^{1-\gamma}}{1-\gamma}+\Phi_{2} \exp \{-2 \delta\} \frac{c_{2}^{1-\gamma}}{1-\gamma}+ \\
& +\lambda\left(C_{0}-c_{0}-\exp \{-r\} c_{1}-\exp \{-2 r\} c_{2}\right) \longrightarrow \max
\end{aligned}
$$

which leads to

$$
c_{1}=c_{0} \exp \left\{\frac{r-\delta}{\gamma}\right\} \Phi_{1}^{1 / \gamma} \quad c_{2}=c_{0} \exp \left\{2 \frac{r-\delta}{\gamma}\right\} \Phi_{2}^{1 / \gamma}
$$

Generalizing for $\tau_{t}$ subperiods we obtain (if $\gamma \neq 0$ and $c_{j}>0 \forall j$ )

$$
C_{0}=c_{0} \sum_{j=0}^{\tau_{t}-1} \Phi_{j}{ }^{1 / \gamma} \exp \left\{\frac{j(r(1-\gamma)-\delta)}{\gamma}\right\} \quad\left(\Phi_{0}=1\right)
$$

Based on this result we can reformulate the model such that optimal consumption 
in the subperiods is taken into account. This is accomplished without increasing the number of decision variables. We replace the original decision variables $C_{0}$ and $\tilde{C}_{t}$ (which refer to consumption in an entire period) by the annual consumption variables $c_{0}$ and $\tilde{c}_{t}$. We define

$$
\alpha_{t j}=\exp \left\{\frac{r-\delta}{\gamma}\right\} \Phi\left(y_{t}, j\right)^{1 / \gamma}
$$

and

$$
\alpha_{t}=\sum_{j=0}^{\tau_{t}-1} \Phi\left(y_{t}, j\right)^{1 / \gamma} \exp \left\{\frac{j(r(1-\gamma)-\delta)}{\gamma}\right\}=\sum_{j=0}^{\tau_{t}-1} \alpha_{t j} \exp \{-j r\}
$$

The budget constraints are now formulated as

$$
\begin{aligned}
& \alpha_{0} c_{0}+\sum_{i=1}^{N} P_{0}^{i}\left(1+q_{p}^{i}\right)=\sum_{i=1}^{N} S_{0}^{i}\left(1-q_{s}^{i}\right)+L_{0}+F_{0} \\
& \alpha_{t} \tilde{c}_{t}+\sum_{i=1}^{N} \tilde{P}_{t}^{i}\left(1+q_{p}^{i}\right)=\sum_{i=1}^{N} \tilde{S}_{t}^{i}\left(1-q_{s}^{i}\right)+L_{t}+F_{t} \quad t=1, \ldots, T-1 .
\end{aligned}
$$

Utility of consumption in $t$ is formulated in terms of $\tilde{c}_{t}$

$$
U\left(\tilde{C}_{t}\right)=\sum_{j=0}^{\tau_{t}-1} \Phi\left(y_{t}, j\right) \exp \{-j \delta\} U\left(\tilde{c}_{t} \alpha_{j t}\right)
$$

A similar consideration relates to the utility of bequest. It is not reasonable to assume that death only occurs at the end of a long time interval. However, to adjust the utility of bequest accordingly is not as straightforward as in the case of consumption. In particular, it is not clear which return should be used for discounting wealth. In addition, preliminary analyses indicate that an approach similar to the one used for consumption does not lead to a closed-form solution. Therefore we leave this issue to future research. 


\subsection{Choice of breakpoints}

The linearization of the objective function requires choosing the number and the position of breakpoints. This choice affects the standard errors of the SLP optimization results as shown in Section 3. We define separate breakpoints for consumption and bequest to account for the different orders of magnitude of the two variables. In addition, these variables may show considerable variation across stages which requires using different breakpoints for each stage, too.

To define the minimum and maximum breakpoints for consumption we use closedform solutions from Ingersoll (1987, p. 238, 242) and Duffie (1996, p. 198) as a guideline. To determine minimum and maximum breakpoints of bequest we consider a simplified version of the problem. For all nodes of a specific stage we assume that the fraction of consumed wealth and the asset allocation is the same. We use the same returns that are subsequently used to solve the SLP. Then we define a random grid of consumptionwealth ratios and asset allocations which obey leverage constraints and other bounds. We evaluate the objective function for each element of the grid, whereby we can use the exact form of the utility function. The optimal solution provides a rough guess for the order of magnitude and the dispersion of consumption and bequest in each stage. This guess is used to define the minimum and maximum breakpoints required for the linearization of the utility function.

To obtain optimal positions of the remaining breakpoints we have considered and tested the following alternatives:

1. minimize the vertical distance between the piecewise linear function and the nonlinear utility function at the midpoints of two adjacent breakpoints,

2. minimize the area between the utility function and the piecewise linear function, or

3. use the curvature of the utility function.

We have found that all three methods yield similar results in terms of the standard 
errors of the solution. In the applications presented in Section 3 we use the curvaturebased approach since it requires no optimization, and it is also much faster than the other methods. The algorithm first divides the interval between $b_{t}^{0}$ and $b_{t}^{m}$ into $n$ equally wide segments separated by the points $\beta_{t}^{j}(j=0, \ldots, n)$ where $\beta_{t}^{0}=b_{t}^{0}$ and $\beta_{t}^{m}=b_{t}^{m}$. The curvature for each $\beta_{t}^{j}$ is defined as (for details see Hanke and Huber 2008)

$$
\kappa_{t}^{j}=\frac{U^{\prime \prime}\left(\beta_{t}^{j}\right)}{\left(1+\left[U^{\prime}\left(\beta_{t}^{j}\right)\right]^{2}\right)^{3 / 2}} .
$$

The average curvature in each segment is the arithmetic mean of two consecutive curvatures

$$
\bar{\kappa}_{t}^{j}=0.5\left(\kappa_{t}^{j-1}+\kappa_{t}^{j}\right) \quad j=1, \ldots, m
$$

The relative average curvature is given by

$$
\hat{\kappa}_{t}^{j}=\frac{\bar{\kappa}_{t}^{j}}{\sum_{j} \bar{\kappa}_{t}^{j}} \quad j=1, \ldots, m
$$

and is used to compute the number of breakpoints in each segment $n_{t}^{j}=\left[m \cdot \hat{\kappa}_{t}^{j}\right]$, where [.] denotes rounding to the nearest integer (surplus breakpoints can be ignored). The position of breakpoints is defined by

$$
b_{t}^{j}=b_{t}^{j-1}+\left(\beta_{t}^{j+1}-\beta_{t}^{j}\right) / n_{t}^{j} \quad j=1, \ldots, m-1 .
$$

\subsection{Scenario generation and choice of intervals}

The uncertainty associated with the consumption-investment problem is modelled by a $K$-dimensional random process. The multivariate return process evolves in discrete time, and the underlying probability distributions are approximated by discrete distri- 
butions in terms of a scenario tree. We follow the approach by Høyland and Wallace (2001), Høyland et al. (2003) and Kaut (2003) to match the first four moments (including the correlations) of the simulated processes. For a $K$-dimensional process a branching factor of $2 K$ is necessary to match the first four (co)moments within reasonable time. More nodes facilitate the matching of moments but increase the number of scenarios. In the examples presented in Section 3 we set $n_{t}=2 K+2, \forall t$. To avoid arbitrage opportunities in the simulated returns (which would be exploited by the optimization algorithm) we apply the procedure proposed by Klaassen (2002).

The number of scenarios in the tree grows exponentially with the number of stages (at which decisions are made) and the number of scenarios following each node. Given the long period of time covered by a life-cycle model, it is computationally infeasible to work with annual decision (rebalancing) intervals over the entire lifetime of an investor. To keep the total number of scenarios practically manageable (e.g. several thousand scenarios) only a rather small number of stages (e.g. three to six) and a small number of nodes is usually considered. For example, in Dempster et al. (2003) the first revision of the portfolio is made after one year since the initial decisions are considered to be most important. The remaining time intervals are much longer and serve to approximate the fact that further portfolio revisions are possible until the planning horizon is reached. This approach implies that the investor is 'locked in' in the chosen asset allocation for a considerable amount of time - possibly much longer than the planned or anticipated rebalancing interval. This problem can be partly alleviated by using more stages and shorter time intervals, but more scenarios and longer solution times would be required.

Therefore we consider a different approach which consists of a sequence of oneyear periods followed by a long, steady-state period which lasts until the maximum lifetime of the investor. This design accounts for the possibility that external cash flows in the near future can be anticipated reasonably well or are even known exactly. Thus, the effect of those cash flows on portfolio and rebalancing decisions in the first few years is taken into account. We use the analytical solution obtained by Richard 
(1975) to derive the utility from optimal consumption and investment decisions in the steady-state period. This amounts to reformulate the objective function as follows:

$$
U\left(C_{0}\right)+E\left[\sum_{t=1}^{T-1} \Theta_{t} U\left(\tilde{B}_{t}\right) D_{t}+\sum_{t=1}^{T-2} \Lambda_{t} U\left(\tilde{C}_{t}\right) D_{t}+\Lambda_{T-1} J\left(\tilde{W}_{T-1}^{+}, y_{T-1}\right) D_{T-1}\right] \longrightarrow \max
$$

$J\left(\tilde{W}_{T-1}^{+}, y_{T-1}\right)$ is the value function (2) defined in Appendix A. It depends on available wealth $\tilde{W}_{T-1}^{+}$(which includes the present value of future labor income or other cash flows) and the age of the investor $y_{T-1}$ at the beginning of the steady-state period. As described in Appendix A the value function is derived in a continuous-time setting. It accounts for optimal consumption and trading, the investor's survival probability, and it is based on geometric Brownian motions for the risky assets and power utility. To implement the steady-state solution according to Richard (1975) we need to define the tangency portfolio. To be consistent with his continuous-time setting the means of the assets are defined as $\boldsymbol{\mu}+0.5 \operatorname{diag}(\mathbf{C})$ (where $\boldsymbol{\mu}$ is the vector of mean log returns and $\mathbf{C}$ is their covariance matrix). An asset which earns the risk-free rate is added to the set of traded assets. Its (constant) return $r$ is also included in the check for arbitrage opportunities in the scenario generation.

Using analytical results from a continuous-time framework in the discrete-time optimization model has obvious advantages. We avoid the unrealistic implications associated with long rebalancing intervals, and we can reduce the number of stages and the size of the scenario tree. It has to be admitted, however, that the value function does not account for restrictions on asset weights or transaction costs. There is also an inconsistency associated with combining one-year decision intervals in discrete time with continuous consumption and trading. In our opinion, however, the advantages outweigh these drawbacks by far. 


\section{$3 \quad$ Numerical results}

To test the scenario generation and the SLP formulation we first present results from cases where closed-form solutions are available. Thereafter we consider life-cycle consumption and asset allocation decisions in settings which include cash flows and labor income, and we investigate the effects associated with long rebalancing intervals.

Closed-form solutions are obtained from Ingersoll (1987), Duffie (1996) and Richard (1975) (see Appendix A) and compared to the SLP-based results in terms of consumption and asset allocation decisions. In case of uncertain lifetime we use survival probabilities for Austrian men estimated in 2005. For cases with certain lifetime the survival probabilities $\varphi$ are set to 1 (except for the final one). We consider two risky assets with a drift rate of 0.06 and a risk-free asset with a return of 0.04 . The correlation among risky assets is 0.5 and their volatility is $0.2 .^{2}$ The node structures considered are $6 \times 6$ and $36 \times 12$, and the linearization of the objective is based on 40 and 80 breakpoints, respectively. We present results for stage $t=0$ in terms of means and standard errors from sampling and solving the model 100 times. The same 100 scenario trees are used in each setting (e.g. for varying risk aversion or time preference). We consider small-scale problems with only a few scenarios (36 and 432, respectively) which can be solved in only a few seconds. By solving such problems repeatedly we can quickly obtain standard errors of the optimal solutions. This is preferable to solving large-scale problems once, which provide better but unknown precision.

Table 1 shows that closed-form results can be replicated with relatively high precision even if only a few scenarios are used. The standard errors for optimal consumption are negligibly small. The slight discrepancies found for optimal consumption may be explained by our combination of the discrete-time SLP structure with the steady-state solution (which implies continuous consumption and rebalancing). The precision of portfolio weights is lower than for consumption. However, the accuracy of the results

\footnotetext{
${ }^{2}$ This choice yields an equally weighted portfolio for an investor with log utility. With power utility and $\gamma>1$ the weights of the risky assets are reduced by the factor $1 / \gamma$.
} 
can be improved by increasing the number of scenarios, and/or using more breakpoints in the linearization of the utility function.

Given that closed-form solutions can be replicated very well we now analyze optimal consumption and asset allocation over the life-cycle using assumptions where closedform solutions are not available. The results in the rest of this section are based on scenario trees with four stages, starting with three one-year periods, and followed by a long period which lasts until the maximum age of 101 years. The node structure is $6 \times 6 \times 6$ which amounts to 216 scenarios. The linearization of the objective function is based on the curvature approach using 40 breakpoints. We use the same set of assets (with the same stochastic properties) as in Table 1.

We first consider the effect of fixed cash in- and outflows on consumption-investment decisions. Cash flows equal to $\pm 0.6 w_{0}$ and $\pm w_{0}$ are assumed to take place in $t=1$ or $t=3$. We take transaction costs of $0.5 \%$ for purchases and sales into account. Initial wealth $w_{0}$ is held only in asset A. Table 2 shows a variety of interesting effects. As expected, the larger the cash flow, the stronger the impact on consumption and investment (compared to the case of zero cash flows). However, consumption does not respond symmetrically to in- and outflows. Outflows occuring at later stages allow for more consumption in $t=0$, whereas the timing of inflows does not affect consumption. This can be explained by the constraint that total wealth has to remain positive under all circumstances. The share invested in risky assets can be higher if outflows are postponed. The reverse holds for inflows. We also note that the share of asset A exceeds the share of asset B in all cases. Since initial wealth is only invested in A, transactions costs prevent equal weights of the two risky assets.

Bodie et al. (1992) and Chen et al. (2006) find a significant impact of human capital on asset allocation decisions over the life cycle. They consider cases where initial labor income is between $10 \%$ and $30 \%$ of initial wealth, and their results are characterized by extreme short positions in the risk-free asset. We have replicated their results to the extent possible given the differences in the two settings. We also 
investigate the importance of labor income on consumption and investment decisions, but in addition we take mortality risk into account. As opposed to their models we treat labor income as deterministic to make use of Richard's (1975) closed-form solution. However, the uncertainty associated with survival probabilities is accounted for as described in equation (1).

Table 3 shows optimal consumption and asset weights for various assumptions about labor income $\left(L_{0}=5,10\right.$ and 15$)$. We find that consumption increases with both labor income and age. This can be explained by the hedging effect associated with the certain stream of income. As expected, accounting for mortality risk has little impact on consumption for relatively young investors. Older investors who take mortality risk into account consume more, and this effect increases with income. In agreement with Bodie et al. (1992) we also find a distinct age effect: The share of risky assets decreases with age. The hedging effect leads to an increase in the share of risky assets as labor income increases. Accounting for mortality risk does not have a consistent effect on the asset allocation. It seems to depend on the level of income and age but the patterns are difficult to explain.

In Section 2.6 we have argued that choosing rather long time intervals between stages may distort results compared to the case of frequent rebalancing. In Table 4 we show results for different rebalancing intervals. We compare solutions which are based on adjusting utility for optimal subperiod consumption as derived in Section 2.4 (columns labelled 'yes') to those where utility of consumption is only adjusted for the length of the interval (i.e. $\alpha_{t}=\tau_{t}$ ). In general, we find that discrepancies between the two versions become only apparent if very long intervals are considered. In particular, the adjusted annual consumption is very stable across intervals which indicates that the adjustment has the intended effect. Except for 20-year intervals, both versions produce almost the same asset allocation, and the investment in risky assets slightly drops with the length of the interval. The strong discrepancy between the two versions for 20-year intervals is hard to explain. Overall, the investment results indicate the 
presence of further effects associated with long rebalancing intervals which are not yet accounted for (e.g. death only occurs at the end of a period; there is no possibility to rebalance the portfolio during a long holding period, which leads to a reduction in the share of risky assets). As already indicated in Section 2.4, however, the necessary adjustments are not straightforward and are left to future research. Unless this issue has been solved satisfactorily, these results suggest that very long rebalancing intervals should rather not be used.

\section{Conclusion}

We have presented a stochastic linear programming approach to obtain optimal consumption and life-cycle asset allocation of an investor with uncertain lifetime. We have first shown that available closed-form solutions can be accurately replicated with the SLP-based approach. Key requirements are exactly matching the moments of the distributions of asset returns, and the linearization of the utility function. We use a discrete scenario tree with only a few stages. To cover the very long time span required in a life-cycle context we suggest working with a few one-year periods followed by a long, steady-state period. We find that the SLP approach is a flexible tool that may be used to assess the importance of aspects such as mortality risk, external cash flows, transaction costs, short-sale constraints, and labor income.

\section{Appendix}

\section{A Closed-form solutions in case of uncertain lifetime}


Richard (1975) obtains a closed-form solution for the consumption and investment decisions of an uncertain lived investor in a continuous time model. He assumes geometric Brownian motions for the risky assets, one riskless asset, and power utility for consumption and bequest of an investor whose current age is $y_{t}$. Provided that relative risk aversion $\gamma$ is the same in both utility functions, the closed-form solution for the value function $J$ is given by

$$
J\left(W_{t}, y_{t}\right)=\frac{a_{y_{t}}}{1-\gamma}\left(W_{t}+H_{t}\right)^{1-\gamma}
$$

The value function is based on the following definitions:

$$
a_{y_{t}}=\left(\int_{y_{t}}^{\bar{\tau}} k(\theta) \frac{S(\theta)}{S\left(y_{t}\right)} \exp \left\{\frac{1-\gamma}{\gamma}(v+r)\left(\theta-y_{t}\right)\right\} d \theta\right)^{\gamma}
$$

with

$$
k(\theta)=[h(\theta) m(\theta)]^{1 / \gamma}+m(\theta)^{1 / \gamma} \quad m(\theta)=\exp \left\{-\delta\left(\theta-y_{t}\right)\right\} \quad v=\frac{\left(\nu_{p}-r\right)^{2}}{2 \gamma \sigma_{p}^{2}} .
$$

$\nu_{p}$ and $\sigma_{p}$ are drift and standard deviation of the tangency portfolio (which only consists of risky assets). $S\left(y_{t}\right)$ is the survival function defined as

$$
S\left(y_{t}\right)=P\left(\theta \geq y_{t}\right)=\int_{y_{t}}^{\bar{\tau}} \vartheta(\theta) d \theta \quad \int_{0}^{\bar{\tau}} \vartheta(\theta) d \theta=1
$$

$h(\theta)$ is the conditional probability density for death conditional upon the investor being alive at age $\theta$, so that $h(\theta)=\vartheta(\theta) / S(\theta)$.

$H_{t}$ is the present value of labor income received until the final age of the underlying mortality table $\bar{\tau}=101 . H_{t}$ assumes an actuarially fair life insurance of labor income and is given by

$$
H_{t}=\int_{y_{t}}^{\bar{\tau}} \mathcal{L}(s) \frac{S(s)}{S\left(y_{t}\right)} \exp \left\{-\left(s-y_{t}\right) r\right\} d s
$$


where $\mathcal{L}(s)$ is continuous labor income and $S(s) / S\left(y_{t}\right)$ is the conditional probability density to be alive at time $s$ conditional upon the investor being alive at age $y_{t}$. This definition of $H_{t}$ agrees with the continuous-time formulation of Richard (1975). The results presented in Section 3 are based on the discrete-time version of labor income defined in Section 2.2, equation (1).

Since we work with discrete mortality tables where age is integer-valued we can simplify the definition of $a_{y_{t}}$ as follows:

$$
\begin{aligned}
& a_{y_{t}}=\left(\sum_{\theta=y_{t}}^{\bar{\tau}-1} k(\theta) \frac{S(\theta)}{S\left(y_{t}\right)} \int_{\theta}^{\theta+1} \exp \left\{c\left(u-y_{t}\right)\right\} d u\right)^{\gamma} \quad c=\frac{1-\gamma}{\gamma}(v+r) \\
& a_{y_{t}}=\left(\sum_{\theta=y_{t}}^{\bar{\tau}-1} k(\theta) \frac{S(\theta)}{S\left(y_{t}\right)}\left[\exp \left\{c\left(\theta-y_{t}\right)\right\}(\exp \{c\}-1) / c\right]\right)^{\gamma} .
\end{aligned}
$$




\section{References}

Barberis, N. C. (2000). Investing for the long run when returns are predictable. Journal of Finance, 55:225-264.

Birge, J. R. and Louveaux, F. (1997). Introduction to Stochastic Programming. Springer.

Bodie, Z., Merton, R. C., and Samuelson, W. F. (1992). Labor supply flexibility and portfolio choice in a life cycle model. Journal of Economic Dynamics and Control, $16: 427-449$.

Brandt, M. W., Goyal, A., Santa-Clara, P., and Stroud, J. R. (2005). A simulation approach to dynamic portfolio choice with an application to learning about return predictability. The Review of Financial Studies, 18(3):831-873.

Brennan, M. J., Schwartz, E. S., and Lagnado, R. (1997). Strategic asset allocation. Journal of Economic Dynamics and Control, 21:1377-1403.

Campbell, J. Y., Chan, Y. L., and Viceira, L. M. (2003). A multivariate model of strategic asset allocation. Journal of Financial Economics, 67:41-80.

Cariño, D. R., Myers, D. H., and Ziemba, W. T. (1998). Concepts, technical issues, and uses of the Russell-Yasuda-Kasai financial planning model. Operations Research, 46:450-462.

Chen, P., Ibbotson, R. G., Milevsky, M. A., and Zhu, K. X. (2006). Human capital, asset allocation, and life insurance. Financial Analysts Journal, 62:97-109.

Cocco, J. F., Gomes, F. J., and Maenhout, P. J. (2005). Consumption and portfolio choice over the life cycle. Review of Financial Studies, 18 (2):491-533.

Dempster, M. A. H., Germano, M., Medova, E. A., and Villaverde, M. (2003). Global asset liability management. British Actuarial Journal, 9:137-216.

Detemple, J. B., Garcia, R., and Rindisbacher, M. (2003). A Monte Carlo method for optimal portfolios. Journal of Finance, 58:401-446. 
Duffie, D. (1996). Dynamic Asset Pricing Theory. Princeton University Press.

Gassmann, H. I. and Schweitzer, E. (2001). A comprehensive input format for stochastic linear programs. Annals of Operations Research, 104(1-4):89-125.

Geyer, A. and Ziemba, W. (2007). The Innovest Austrian pension fund financial planning model InnoALM. forthcoming in Operations Research.

Gomes, F. and Michaelides, A. (2005). Optimal life-cycle asset allocation: Understanding the empirical evidence. Journal of Finance, 60:869-904.

Hanke, M. and Huber, S. (2008). Curvature, not second derivative. Mathematical Spectrum. forthcoming.

Høyland, K., Kaut, M., and Wallace, S. W. (2003). A heuristic for moment-matching scenario generation. Computational Optimization and Applications, 24:169-185.

Høyland, K. and Wallace, S. W. (2001). Generating scenario trees for multistage decision problems. Management Science, 47:295-307.

Ingersoll, J. E. (1987). Theory of financial decision making. Rowman \& Littlefield.

Kaut, M. (2003). Updates to the published version of 'A Heuristic for Momentmatching Scenario Generation by K.Høyland, M. Kaut, and S.W. Wallace'. working paper.

King, A. J. (2002). Duality and martingales: a stochastic programming perspective on contingent claims. Mathematical Programming Series B, 91:543-562.

King, A. J., Wright, S. E., Parija, G. R., and Entriken, R. (2005). Applications of Stochastic Programming, chapter The IBM Stochastic Programming System, pages 21-37. MPS SIAM - Series in Optimization.

Klaassen, P. (2002). Comment on 'Generating Scenario Trees for Multistage Decision Problems'. Management Science, 48:1512-1516.

Longstaff, F. and Schwartz, E. (2001). Valuing American options by simulation: A simple least-square approach. Review of Financial Studies, 14 (1):113-147. 
Merton, R. C. (1969). Lifetime portfolio selection under uncertainty: the continuoustime case. Review of Economics and Statistics, 51:247-257.

Merton, R. C. (1971). Optimum consumption and portfolio rules in a continuous-time model. Journal of Economic Theory, 3:373-413.

Richard, S. F. (1975). Optimal consumption, portfolio and life insurance rules for an uncertain lived individual in a continous time model. Journal of Financial Economics, 2:187-203.

Samuelson, P. A. (1969). Lifetime portfolio selection by dynamic stochastic programming. Review of Economics and Statistics, 51:239-246.

Schroder, M. and Skiadas, C. (1999). Optimal consumption and portfolio selection with stochastic differential utility. Journal of Economic Theory, 89 (1):68-126.

Wallace, S. W. and Ziemba, W. T. (2005). Applications of Stochastic Programming. MPS-SIAM Book Series on Optimization.

Zenios, S. A. and Ziemba, W. T. (2006). Handbook of Asset and Liability Management: Theory and Methodology. North-Holland.

Ziemba, W. T. and Mulvey, J. (1998). Worldwide Asset and Liability Modeling. Cambridge University Press. 


\begin{tabular}{lcccc}
\hline \multicolumn{1}{l}{ consumption } & asset A & asset B & risk-free \\
\hline \hline log utility, $d=1$, certain lifetime & & & & \\
\hline closed-form solution & 1.61 & 33.33 & 33.33 & 33.33 \\
40 breakpoints, $6 \times 6$ nodes & 1.65 & 31.80 & 31.78 & 36.42 \\
& $(0.00)$ & $(0.19)$ & $(0.20)$ & $(0.12)$ \\
80 breakpoints, $6 \times 6$ nodes & 1.63 & 32.86 & 32.80 & 34.34 \\
& $(0.00)$ & $(0.07)$ & $(0.08)$ & $(0.14)$ \\
40 breakpoints, $36 \times 12$ nodes & 1.64 & 32.97 & 32.99 & 34.04 \\
& $(0.00)$ & $(0.07)$ & $(0.06)$ & $(0.05)$ \\
80 breakpoints, $36 \times 12$ nodes & 1.63 & 33.01 & 32.98 & 34.01 \\
& $(0.00)$ & $(0.04)$ & $(0.04)$ & $(0.02)$ \\
\hline log utility, $d=0.96$, certain lifetime & & & \\
\hline closed-form solution & 4.35 & 33.33 & 33.33 & 33.33 \\
40 breakpoints, $6 \times 6$ nodes & 4.27 & 32.94 & 33.03 & 34.03 \\
& $(0.00)$ & $(0.09)$ & $(0.08)$ & $(0.11)$ \\
80 breakpoints, $6 \times 6$ nodes & 4.37 & 34.60 & 34.72 & 30.67 \\
& $(0.00)$ & $(0.22)$ & $(0.21)$ & $(0.42)$ \\
40 breakpoints, $36 \times 12$ nodes & 4.36 & 33.04 & 33.01 & 33.95 \\
& $(0.00)$ & $(0.05)$ & $(0.06)$ & $(0.04)$ \\
80 breakpoints, $36 \times 12$ nodes & 4.36 & 33.00 & 33.01 & 33.99 \\
& $(0.00)$ & $(0.04)$ & $(0.04)$ & $(0.02)$ \\
\hline log utility, $d=0.96$, uncertain lifetime & & & \\
\hline closed-form solution & 5.47 & 33.33 & 33.33 & 33.33 \\
40 breakpoints, $6 \times 6$ nodes & 5.40 & 33.38 & 33.38 & 33.25 \\
& $(0.00)$ & $(0.03)$ & $(0.05)$ & $(0.05)$ \\
80 breakpoints, $6 \times 6$ nodes & 5.40 & 32.68 & 32.85 & 34.47 \\
& $(0.00)$ & $(0.06)$ & $(0.05)$ & $(0.08)$ \\
40 breakpoints, $36 \times 12$ nodes & 5.40 & 33.05 & 33.03 & 33.92 \\
& $(0.00)$ & $(0.05)$ & $(0.04)$ & $(0.04)$ \\
80 breakpoints, $36 \times 12$ nodes & 5.39 & 33.01 & 32.98 & 34.01 \\
& $(0.00)$ & $(0.03)$ & $(0.03)$ & $(0.02)$ \\
\hline power utility, $\gamma=4, d=0.96$, certain lifetime & & & \\
\hline closed-form solution & 4.30 & 8.33 & 8.33 & 83.33 \\
40 breakpoints, $6 \times 6$ nodes & 4.47 & 8.83 & 8.55 & 82.61 \\
& $(0.00)$ & $(0.12)$ & $(0.11)$ & $(0.14)$ \\
& 4.46 & 8.80 & 8.61 & 82.59 \\
& $(0.00)$ & $(0.07)$ & $(0.05)$ & $(0.11)$ \\
& 4.48 & 8.27 & 8.20 & 83.54 \\
& $(0.00)$ & $(0.03)$ & $(0.03)$ & $(0.03)$ \\
& 4.46 & 8.24 & 8.26 & 83.50 \\
& $(0.00)$ & $(0.02)$ & $(0.02)$ & $(0.02)$ \\
\hline
\end{tabular}

Table 1: Optimal consumption and asset allocation from closed-from solutions and the stochastic linear program in $t=0$. Results are presented in terms of means and standard errors (in parentheses) from 100 solutions of the problem. The same 100 scenario trees are used for each entry of the table. The investor is assumed to be 40 years old. $d$ is the time discount factor. For cases with uncertain lifetime we use Austrian mortality tables for men. 


\begin{tabular}{cccccc}
\hline cash flow & stage & consumption & asset A & asset B & risk-free \\
\hline 0 & & 4.3 & 10.2 & 6.0 & 83.8 \\
& & $(0.00)$ & $(0.04)$ & $(0.03)$ & $(0.04)$ \\
\hline-60 & $t=1$ & 1.8 & 4.3 & 2.2 & 93.5 \\
& & $(0.00)$ & $(0.01)$ & $(0.02)$ & $(0.02)$ \\
& $t=3$ & 2.1 & 4.8 & 2.3 & 92.9 \\
& & $(0.00)$ & $(0.02)$ & $(0.02)$ & $(0.03)$ \\
\hline-100 & $t=1$ & 0.15 & 0.40 & 0.14 & 99.5 \\
& & $(0.00)$ & $(0.00)$ & $(0.01)$ & $(0.01)$ \\
& $t=3$ & 0.47 & 1.07 & 0.61 & 98.3 \\
+60 & $t=1$ & $(0.00)$ & $(0.01)$ & $(0.00)$ & $(0.01)$ \\
\hline & & $(0.8$ & 17.0 & 9.7 & 73.3 \\
& $t=3$ & 6.8 & $(0.11)$ & $(0.08)$ & $(0.18)$ \\
& & $(0.00)$ & $(0.05)$ & $(0.07)$ & $(0.08)$ \\
\hline+100 & $t=1$ & 8.4 & 23.9 & 11.2 & 64.9 \\
& & $(0.01)$ & $(0.08)$ & $(0.14)$ & $(0.13)$ \\
& $t=3$ & 8.3 & 22.6 & 10.4 & 66.9 \\
& & $(0.00)$ & $(0.00)$ & $(0.00)$ & $(0.00)$ \\
\hline
\end{tabular}

Table 2: Optimal consumption and asset allocation for various assumptions about current, annual labor income $L_{0}$. Initial wealth is $w_{0}=100$. Results are presented in terms of means and standard errors (in parentheses) from 100 solutions of the problem. We consider a man at age 40 with power utility $(\gamma=4)$ and time discount factor $d=0.96$. 


\begin{tabular}{|c|c|c|c|c|c|c|c|c|c|}
\hline \multirow[b]{2}{*}{ income } & \multirow[b]{2}{*}{ age } & \multicolumn{2}{|c|}{ consumption } & \multicolumn{2}{|c|}{ asset $\mathrm{A}$} & \multicolumn{2}{|c|}{ asset B } & \multicolumn{2}{|c|}{ risk-free } \\
\hline & & $\mathrm{u}$ & $\mathrm{c}$ & $\mathrm{u}$ & $\mathrm{c}$ & $\mathrm{u}$ & $\mathrm{c}$ & $\mathrm{u}$ & $\mathrm{c}$ \\
\hline \multirow{6}{*}{$L_{0}=5$} & 40 & 9.2 & 9.4 & 16.9 & 17.3 & 17.1 & 17.2 & 65.9 & 65.5 \\
\hline & & $(0.00)$ & $(0.00)$ & $(0.24)$ & $(0.09)$ & $(0.24)$ & $(0.09)$ & $(0.45)$ & $(0.17)$ \\
\hline & 60 & 11.5 & 9.1 & 15.5 & 17.3 & 15.4 & 17.4 & 69.1 & 65.3 \\
\hline & & $(0.01)$ & $(0.00)$ & $(0.12)$ & $(0.08)$ & $(0.10)$ & $(0.08)$ & $(0.17)$ & $(0.15)$ \\
\hline & 80 & 17.8 & 11.4 & 14.7 & 14.1 & 14.6 & 13.8 & 70.7 & 72.1 \\
\hline & & $(0.00)$ & $(0.00)$ & $(0.17)$ & $(0.15)$ & $(0.16)$ & $(0.17)$ & $(0.28)$ & $(0.28)$ \\
\hline \multirow{6}{*}{$L_{0}=10$} & 40 & 12.0 & 12.7 & 25.2 & 27.1 & 25.4 & 27.0 & 49.4 & 45.9 \\
\hline & & $(0.00)$ & $(0.00)$ & $(0.29)$ & $(0.18)$ & $(0.32)$ & $(0.21)$ & $(0.58)$ & $(0.25)$ \\
\hline & 60 & 17.1 & 11.9 & 24.8 & 25.4 & 25.1 & 25.2 & 50.1 & 49.3 \\
\hline & & $(0.00)$ & $(0.00)$ & $(0.27)$ & $(0.14)$ & $(0.26)$ & $(0.15)$ & $(0.43)$ & $(0.21)$ \\
\hline & 80 & 24.9 & 16.2 & 20.2 & 19.9 & 20.0 & 19.9 & 59.9 & 60.2 \\
\hline & & $(0.01)$ & $(0.00)$ & $(0.12)$ & $(0.13)$ & $(0.14)$ & $(0.13)$ & $(0.20)$ & $(0.26)$ \\
\hline \multirow{6}{*}{$L_{0}=20$} & 40 & 13.5 & 13.6 & 34.5 & 32.6 & 35.0 & 31.4 & 30.5 & 36.0 \\
\hline & & $(0.00)$ & $(0.00)$ & $(0.43)$ & $(0.45)$ & $(0.39)$ & $(0.45)$ & $(0.72)$ & $(0.68)$ \\
\hline & 60 & 19.6 & 14.8 & 32.2 & 33.0 & 32.2 & 32.4 & 35.6 & 34.6 \\
\hline & & $(0.00)$ & $(0.00)$ & $(0.19)$ & $(0.30)$ & $(0.18)$ & $(0.31)$ & $(0.23)$ & $(0.52)$ \\
\hline & 80 & 31.8 & 19.5 & 26.9 & 24.8 & 26.9 & 25.2 & 46.2 & 50.0 \\
\hline & & $(0.00)$ & $(0.00)$ & $(0.22)$ & $(0.16)$ & $(0.21)$ & $(0.19)$ & $(0.26)$ & $(0.18)$ \\
\hline
\end{tabular}

Table 3: Optimal consumption and asset allocation for various assumptions about current, annual labor income $L_{0}$. Initial wealth is $w_{0}=100$. Results are presented in terms of means and standard errors (in parentheses) from 100 solutions of the problem. We consider a man with power utility $(\gamma=4)$ and time discount factor $d=0.96$. Columns labelled with 'u' (uncertain lifetime) indicate that mortality risk is taken into account. Columns labelled with 'c' (certain lifetime) ignore mortality risk. 


\begin{tabular}{|c|c|c|c|c|c|c|c|c|}
\hline \multirow[b]{2}{*}{ intervals } & \multicolumn{2}{|c|}{ consumption } & \multicolumn{2}{|c|}{ asset $\mathrm{A}$} & \multicolumn{2}{|c|}{ asset B } & \multicolumn{2}{|c|}{ risk-free } \\
\hline & yes & no & yes & no & yes & no & yes & no \\
\hline $1-1-1$ & \multicolumn{2}{|c|}{$\begin{array}{c}4.4 \\
(0.00)\end{array}$} & \multicolumn{2}{|c|}{$\begin{array}{c}7.5 \\
(0.08)\end{array}$} & \multicolumn{2}{|c|}{$\begin{array}{c}7.5 \\
(0.08)\end{array}$} & \multicolumn{2}{|c|}{$\begin{array}{c}85.1 \\
(0.14)\end{array}$} \\
\hline $5-5-5$ & $\begin{array}{c}4.7 \\
(0.00)\end{array}$ & $\begin{array}{c}4.3 \\
(0.00)\end{array}$ & $\begin{array}{c}8.1 \\
(0.08)\end{array}$ & $\begin{array}{c}8.0 \\
(0.07)\end{array}$ & $\begin{array}{c}8.1 \\
(0.08)\end{array}$ & $\begin{array}{c}8.0 \\
(0.07)\end{array}$ & $\begin{array}{l}83.7 \\
(0.15)\end{array}$ & $\begin{array}{c}84.0 \\
(0.13)\end{array}$ \\
\hline $10-10-10$ & $\begin{array}{c}4.8 \\
(0.00)\end{array}$ & $\begin{array}{c}4.1 \\
(0.00)\end{array}$ & $\begin{array}{c}7.5 \\
(0.11)\end{array}$ & $\begin{array}{c}7.6 \\
(0.12)\end{array}$ & $\begin{array}{c}7.6 \\
(0.09)\end{array}$ & $\begin{array}{c}7.5 \\
(0.11)\end{array}$ & $\begin{array}{c}84.9 \\
(0.19)\end{array}$ & $\begin{array}{c}84.8 \\
(0.22)\end{array}$ \\
\hline $20-20-20$ & $\begin{array}{c}4.7 \\
(0.00)\end{array}$ & $\begin{array}{c}3.5 \\
(0.00)\end{array}$ & $\begin{array}{c}6.3 \\
(0.12)\end{array}$ & $\begin{array}{c}11.4 \\
(0.24)\end{array}$ & $\begin{array}{c}6.3 \\
(0.12)\end{array}$ & $\begin{array}{l}11.7 \\
(0.20)\end{array}$ & $\begin{array}{c}86.8 \\
(0.22)\end{array}$ & $\begin{array}{l}77.0 \\
(0.41)\end{array}$ \\
\hline
\end{tabular}

Table 4: Optimal consumption and asset allocation in $t=0$ for various rebalancing intervals. Consumption is expressed in annual terms. Results are presented in terms of means and standard errors (in parentheses) from 100 solutions of the problem. We consider a man at age 40 with power utility $(\gamma=4)$ and time discount factor $d=0.96$. The notation $\tau-\tau-\tau$ indicates the use of three rebalancing intervals of length $\tau$, followed by a sufficiently long steady-state period. Columns labelled with 'yes' indicate that the optimal adjustment for subperiod consumption has been taken into account. 'no' refers to no adjustment. 